\section{THU0044 \\ IMPAIRED AUTOPHAGY INDUCES DEFECTIVE FUNCTION OF REGULATORY B CELLS IN ANKYLOSING SPONDYLITIS}

C. Shuizhong, X. zhongyu, Z. guan, S. huiyong, W. yanfeng. Department of Orthopedics, Sun Yat-sen Memorial Hospital, Sun Yat-sen University, China

Background: Ankylosing spondylitis (AS) is an autoimmune disease characterised by pathological osteogenesis and chronic inflammation. Large number studies shown that Regulatory B cells (Bregs) has immunosuppressive function, which could be involved in many rheumatic disease, such as systemic lupus erythematosus (SLE), rheumatoid arthritis (RA). But the Bregs in AS are poorly understood.

Objectives: To investigate the ratio and function of Bregs in AS, and illuminate the under lying mechanism, which might help to further understand the pathology of AS.

Methods: (1) Peripheral blood mononuclear cells (PBMCs) were collected from 9 AS patients and 9 healthy controls, then the Bregs were detected by using flow cytometry with the following antibodies: CD19-PE, CD24-FITC and CD38-APC. ${ }^{2}$ $B$ cells were purified with a CD19 magnetic bead, the Bregs were sorted by using the flow cytometry. Bregs were added to the upper chamber with $1.5 \mathrm{ml}$ medium, while CD4 +T cells were added to the lower chamber with $2.6 \mathrm{ml}$ medium at a ratio of $1: 1$ Bregs $(1 \times 106$ cells $)$ : CD4 +T cells $(1 \times 106$ cells $)$. CD4 +T cells were incubated with $5 \mu \mathrm{M}$ CFDA-SE. The CD4 +T cell proliferation was analyse in the fifth day. ${ }^{3}$ The cytokines of Bregs were detected with a proteome profiler kit, and confirmed by using Elias and Western Blot. ${ }^{4}$ We detected the Bregs autophagy between AS and HD. And we regulate the autophagy with rapamycin and 3-methyladenine (3-MA), then detected the IL-10 secretion of Bregs and the CD4 +T cell proliferation among groups.

Results: (1) The ratio of Bregs in AS was higher that in healthy group. ${ }^{2}$ Bregs from AS shown a impaired function in suppressed the CD4 +T cell proliferation compared with $\mathrm{HD} .^{3}$ Bregs of AS expressed and secreted less IL-10 than healthy control, and might explain the impaired function of Bregs of $\mathrm{AS}^{4}$ Exogenous $\mathrm{LL}-10$ recovered the immunosuppressive capacity of AS Bregs, whereas exogenous anti-IL-10 antibody reduced the immunosuppressive capacity of HD Bregs. ${ }^{5}$ Autophagy impaired in AS Bregs compared with $\mathrm{HD}$ Bregs. Induce autophagy in AS Bregs could increase the IL-10 secretion and strengthened its immunosuppressive capacity, while 3-MA shown the opposite results.

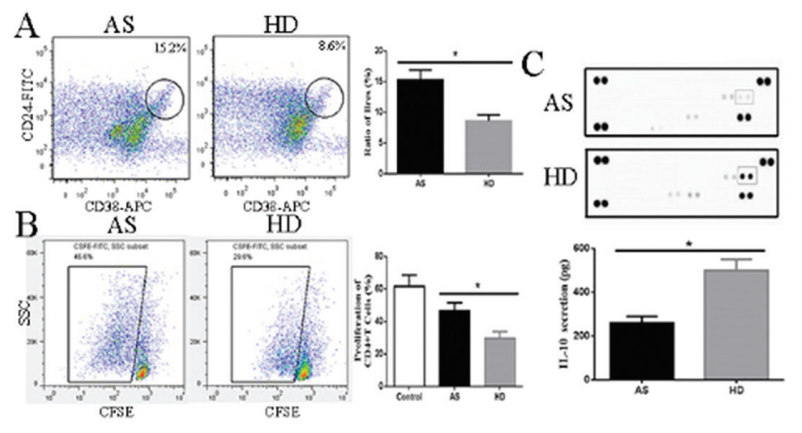

Conclusions: Even increasing ratio of Bregs in AS, but they had a impaired function in suppressed CD4 +T cell proliferation compared with the HD. We further found that impaired autophagy could induces less IL-10 secretion, which further affected the immunosuppressive capacity of Bregs of AS.

Acknowledgements: This study was supported by the National Natural Science Foundation of China $(81271951,81401850)$, the Science and Technology Project of Guangdong Province (2015 B020228001) and by the Engineering Technology Research Centre for Comprehensive Diagnosis and Treatment of Ankylosing Spondylitis of Guangdong Higher Education Institutes (GCZX-A1301).

Disclosure of Interest: None declared

DOI: 10.1136/annrheumdis-2018-eular.6820

\section{THU0045 \\ EXPANSION OF ACTIVATED CXCR5+ICOS+ TFH CELLS AND PLASMABLASTS INDUCED BY SEASONAL INFLUENZA VACCINE IS IMPAIRED IN ANTI-IL-6R TREATED RHEUMATOID ARTHRITIS PATIENTS}

V.C. Romao $^{1,2}$, A. Agua-Doce ${ }^{3}$, J. Polido-Pereira ${ }^{1,2}$, R. Barros ${ }^{2}$, I.P. Lopes ${ }^{1}$, M. I. Seixas ${ }^{2}$, M.J. Saavedra ${ }^{2}$, E. Sacadura-Leite ${ }^{4}$, H. Rebelo-de-Andrade ${ }^{5,6}$, J. E. Fonseca ${ }^{1,2}$, L. Graca ${ }^{3}{ }^{1}$ Rheumatology Research Unit, Instituto de Medicina Molecular, Faculdade de Medicina da Universidade de Lisboa; ${ }^{2}$ Rheumatology Department, Hospital Santa Maria-CHLN; ${ }^{3}$ Lymphocyte Regulation Lab, Instituto de Medicina Molecular, Faculdade de Medicina da Universidade de Lisboa; ${ }^{4}$ Occupational Medicine Department, Hospital Santa Maria-CHLN; ${ }^{5}$ Departamento de Doenças Infecciosas, Instituto Nacional de Saúde Doutor Ricardo Jorge, IP. ${ }^{6}$ Host-Pathogen Interaction Unit, Research Institute for Medicines (iMed. ULisboa), Faculdade de Farmácia, Universidade de Lisboa, Lisboa, Portugal

Background: T follicular helper (Tfh) cells are essential for the generation of high affinity neutralising antibodies elicited following vaccination and are involved in the pathogenesis of rheumatoid arthritis (RA). Interleukin (IL) -6 has been shown to be critical for Tfh differentiation in mice, while its importance in humans has been less clear, given the lack of adequate in vivo assessment.

Objectives: To investigate the importance of IL-6 for the in vivo differentiation of human Tfh cells, taking advantage of influenza vaccination in patients under antiIL-6R therapy.

Methods: Blood was collected before, 7 and 28 days after vaccination from established RA patients treated with tocilizumab (TCZ, IL-6R blocker), methotrexate (MTX) \pm ther DMARDs and age- and sex-matched healthy donors (HD). We analysed the frequency of $\mathrm{Tfh}\left(\mathrm{CD} 3^{+} \mathrm{CD} 4^{+} \mathrm{CD} 25^{-} \mathrm{Foxp}^{-} \mathrm{CXCR} 5^{+} \mathrm{CD} 45 \mathrm{RO} \mathrm{O}^{+}\right), \mathrm{T}$ follicular regulatory $\left(\mathrm{Tfr}, \mathrm{CD}^{+} \mathrm{CD} 4^{+} \mathrm{CD} 25^{+} \mathrm{Foxp} 3^{+} \mathrm{CXCR} 5^{+}\right)$and $\mathrm{B}$ cell populations at each time point. We used non-parametric tests, deemed significant at $p<0.05$. Results: We included 137 participants (42 TCZ, 42 MTX, 53 HD) with similar age and gender distribution. Patients from the TCZ group had more active and severe disease. At baseline, patients treated with TCZ had higher frequency of Tfh and Thh-Th2-like cells (CXCR3 $\left.{ }^{-} \mathrm{CCR} 6^{-}\right)$and lower frequency of Tfr-Th1-like $\left(\mathrm{CXCR}^{+} \mathrm{CCR}^{-}\right)$and $\mathrm{B}$ cells. Following influenza vaccination, the overall blood Tfh and Tfr populations remained unchanged in all groups. However, as previously reported, there were marked changes in specific subsets at day 7 of HD following vaccination. We found a marked expansion of activated $\mathrm{CXCR} 5^{+} \mathrm{ICOS}^{+}$ Tfh cells at day 7, in HD and MTX-treated patients, but this was impaired in the TCZ group (figure 1). The increase in activated CXCR5 ${ }^{+} I C O S^{+}$Tfh cells was mainly due to a Tfh-Th1-like subpopulation, greatly increased in HD and MTXtreated patients (figure 1). Of note, $\mathrm{CXCR}^{+} \mathrm{CCOS}^{+} \mathrm{Tfh}-\mathrm{Th} 17$-like cells also accumulated in HD but not in RA patients. The proliferative capacity of $\mathrm{CXCR} 5^{+} \mathrm{ICOS}^{+}$ Tfh cells seemed to be partially impaired in patients under IL-6R blockade, that displayed marked reduction of $\mathrm{Ki} 67^{+} \mathrm{CD} 38^{+}$proliferative cells within that compartment (figure 1). Anti-IL-6R treatment also impaired expansion of CD19 $\operatorname{lgD}$ $\mathrm{CD} 27^{+} \mathrm{CD} 38^{\text {hi }}$ plasmablasts following vaccination, when compared with both MTX and $\mathrm{HD}$ groups (figure 1). Changes in $\mathrm{CXCR}^{+} \mathrm{ICOS}^{+} \mathrm{Tfh}$ and plasmablasts were significantly correlated in all groups.
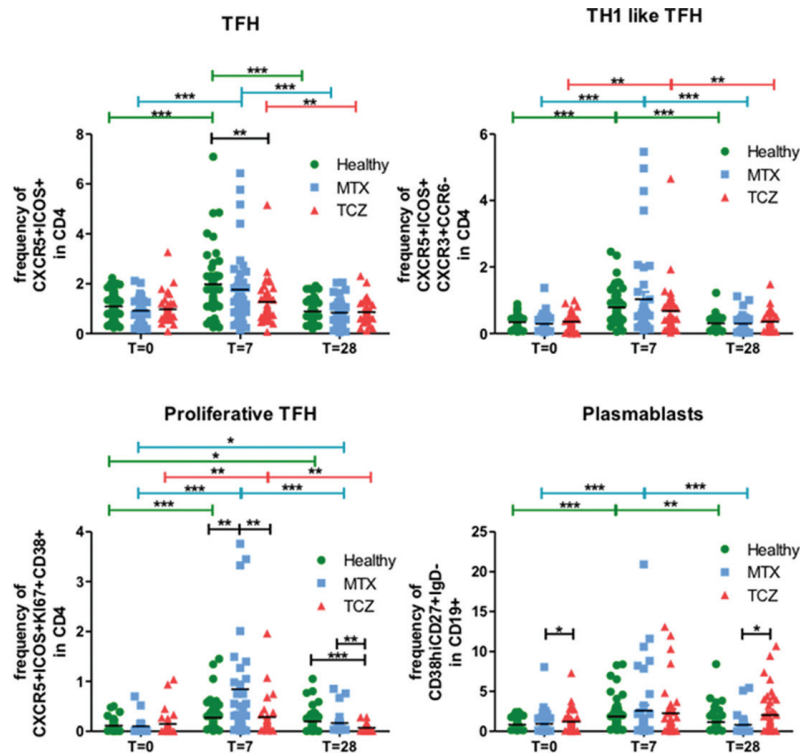

Abstract THU0045 - Figure 1. Frequency of cell populations within the CD4+ in the blood. All tests are paired and non-parametric. Healthy $\mathrm{N}=53, \mathrm{MTX} N=42, \mathrm{TCZ} N=42$. 